\title{
Adverse drug reactions associated with six commonly used antiepileptic drugs in southern China from 2003 to 2015
}

Yanru Du', Jiahe Lin', Jingzan Shen', Siqi Ding ${ }^{1}$, Mengqian Ye ${ }^{1}$, Li Wang ${ }^{1}$, Yi Wang ${ }^{2}$, Xinshi Wang ${ }^{1}$, Niange Xia', Rongyuan Zheng ${ }^{1}$, Hong Chen ${ }^{3}$ and Huiqin $\mathrm{Xu}^{1 *}$

\begin{abstract}
Background: This active, open observational study aimed to investigate adverse drug reactions (ADRs) associated with six commonly used antiepileptic drugs (AEDs) in southern Chinese outpatients with epilepsy from 2003 to 2015.

Methods: The Wenzhou Epilepsy Follow-Up Registry Database (WEFURD) was established by a single epilepsy center in China in January 2003 to record AED efficacy and the associated ADRs by registered outpatients diagnosed with epilepsy. We reviewed the data of outpatients who had only taken one or more of six commonly used AEDs, namely, carbamazepine (CBZ), valproate (VPA), lamotrigine (LTG), oxcarbazepine (OXC), topiramate (TPM) and levetiracetam (LEV), and were registered in the WEFURD between 2003 and 2015. We evaluated the ADRs caused by the single or combined use of the above six specific AEDs based on the WHO-UMC scale. The data of the ADRs were categorized by age, sex, number of AEDs related to ADRs, medications, seriousness of ADRs, causality levels of the WHO-UMC scale and system organ class (SOC). The unit of analysis was one ADR.

Results: A total of 3069 epilepsy outpatients (1807 outpatients with 5049 eligible ADRs and 1262 outpatients without ADRs) were included. The overall ADR rate was 58.88\% (1807/3069). An average of 2.79 ADRs (5049/1807) occurred per patient with an ADR; $53.8 \%$ of the 5049 ADRs were recorded in females, and $50.4 \%$ were caused by monotherapy. Of the ADRs, 10.6\% (537/5049) were severe adverse reactions (SARs), including 34 serious adverse effects (SAEs). The SAR rates caused by one, two and three or more AEDs were $9.9,10.0$ and 19.6\%, respectively $(p<0.001)$. Eighteen SOC categories were identified, and the top three were psychiatric disorders (1633/5049, 32.3\%), neurological disorders (1222/5049, 24.2\%) and gastrointestinal disorders (564/5049, 11.2\%). Of the 537 SARs, skin and appendage disorders accounted for 24.4\% (131/537). Among the 34 SAEs, serious allergies, fetal malformations, renal calculus and pancreatitis accounted for the majority.
\end{abstract}

Conclusions: Our findings suggest that clinicians should pay attention to psychiatric ADRs and be alert for SARs, especially when three or more AEDs are used together. Moreover, active surveillance might provide another method of pharmacovigilance in China.

Keywords: Adverse drug reactions, Antiepileptic drug, Severe adverse reactions, China

\footnotetext{
* Correspondence: xuhuiqin@wmu.edu.cn; xuhuiqin1972@163.com

'Department of Neurology, The First Affiliated Hospital of Wenzhou Medical

University, Shangcai Village, Ouhai District, Wenzhou, Zhejiang Province,

People's Republic of China

Full list of author information is available at the end of the article
}

(c) The Author(s). 2019 Open Access This article is distributed under the terms of the Creative Commons Attribution 4.0 International License (http://creativecommons.org/licenses/by/4.0/), which permits unrestricted use, distribution, and reproduction in any medium, provided you give appropriate credit to the original author(s) and the source, provide a link to the Creative Commons license, and indicate if changes were made. The Creative Commons Public Domain Dedication waiver (http://creativecommons.org/publicdomain/zero/1.0/) applies to the data made available in this article, unless otherwise stated. 


\section{Background}

Adverse drug reactions (ADRs) are a major public health concern [1]. In recent years, the most widely used antiepileptic drugs (AEDs) in our epilepsy center included carbamazepine (CBZ), valproate (VPA), lamotrigine (LTG), oxcarbazepine (OXC), topiramate (TPM) and levetiracetam (LEV). ADRs to AEDs may lead to treatment failure, withdrawal or death [2]. In 2007, an unblinded randomized controlled trial (RCT) was conducted to assess the effectiveness and safety of CBZ, LTG, OXC, or TPM for the treatment of partial epilepsy. The results of this study showed that approximately $50 \%$ of patients reported adverse events [3]. Meta-analyses in the literature showed that AEDs had aggression as a side effect [4], and the behavioral side-effect profiles of AEDs should be considered when choosing optimal AEDs [5]. Furthermore, a recent article reported that the use of aromatic AEDs, e.g., CBZ, OXC and LTG, was more frequently associated with drug hypersensitivity [6]. Attention should also be paid to safety concerns regarding the use of AEDs during pregnancy and breastfeeding [7]. RCTs have improved the quality and reliability of drug evaluation studies, but they have not provided specific clinical safety data [8]. However, for various ethical, statistical and logistical reasons, it is extremely difficult to organize structured clinical studies that are likely to provide substantial data on ADRs [9]. In China, the safety monitoring of AEDs mainly relies on the implementation of the national reporting system of ADRs, which is a spontaneous reporting (SR) system. The reporting of ADRs to the China Food and Drug Administration (CFDA) has been the duty of healthcare professionals. In the SR system, however, the problems of underreporting and poor-quality data are still difficult to solve. Active monitoring from clinicians can compensate for the deficiencies of the SR system, but such research is relatively lacking.

To fill this gap, we conducted a clinical, active, open observational study to investigate the ADRs associated with six commonly used AEDs in epilepsy outpatients in southern China from 2003 to 2015.

\section{Methods}

\section{Data source}

The Epilepsy Long-term Follow Up Registry Study (ELFURS) was a single-center, prospective and observational study of epilepsy outpatients in China. The ELFURS was conducted at the epilepsy center in the First Affiliated Hospital of Wenzhou Medical University (FAHWMU) in January 2003 [10]. The study was approved by the clinical research ethics committee of FAHWMU and registered in the World Health Organization (WHO) Registry Network (registration number: ChiCTR-OCH-14004616). The Wenzhou Epilepsy Follow-Up Registry Database (WEFURD) was established simultaneously to record, save, and process the registry data, which included the demographic information of the epileptic outpatients, the use of AEDs, the efficacy of the AEDs and the AED-related ADRs [10]. An ADR, as defined by the WHO in 1972, is "a response to a drug that is noxious and unintended and occurs at doses normally used in man for the prophylaxis, diagnosis or therapy of disease, or for modification of physiological function" [11]. ADR data were prospectively collected at every visit by questionnaire (every 1-3 months), and relevant information was updated in the following visits. The WEFURD has been maintained by trained two researchers and is the largest epilepsy database in southern China. By January 2018, the WEFURD enrolled 4563 epilepsy outpatients with regular follow-up and included 6259 records of ADRs.

\section{Study protocol}

In this study, we reviewed the data of outpatients who only took one or more of six commonly used AEDs (CBZ, VPA, LTG, OXC, TPM and LEV) and were registered in the WEFURD between 2003 and 2015. We evaluated the ADRs caused by single or combined use of the above six specific AEDs based on the WHOUMC scale [12]. ADRs that were not caused by these six specific AEDs were excluded. ADRs that occurred when these six AEDs were combined with the other AEDs were also excluded. Finally, 1807 epilepsy outpatients with 5049 eligible ADRs and 1262 epilepsy outpatients without ADRs were included in this study. The two groups (epilepsy outpatients with and without ADRs) were compared, and the data of the 5049 ADRs were categorized by age, sex, number of AEDs related to ADRs, medications, severity of ADRs, causality levels of the WHO-UMC scale [12] and system organ class (SOC) according to the WHO Adverse Reactions Terminology (WHOART) [13]. The unit of analysis was one ADR. The preferred terms (PTs) of the WHOART [13] were used to describe and quantify the ADRs.

\section{Validation and classification of ADRs by the WHO-UMC scale}

The WHO-UMC criteria [12] were employed to classify the causality levels as certain, probable/likely, possible, unlikely, conditional/unclassified, and unassessable/ unclassifiable. Prior to assessment, 12 members of the project received unified training. The causality assessment of an ADR was performed independently by two physicians, and this work was completed by eight physicians. The other two physicians checked the consistency of the assessment results. For ADRs with inconsistent assessment results, the two epileptologists (Huiqin $\mathrm{Xu}$ and Rongyuan Zheng) re-evaluated the results together. The ADRs that were still controversial after the reassessment 
process were discussed by all of the team members. We minimized bias from the evaluators through unified training, verification and discussion. The levels of unlikely and unassessable/unclassifiable were excluded in this study.

\section{Classification of ADRs by SOC and severity}

The 5049 ADRs were classified according to the SOCs in the WHOART [13]. Serious adverse effects (SAEs) were defined as those that are lethal or life threatening, require hospital admission or prolongation of an existing hospital stay, result in persistent or significant disability/ incapacity, or are cancers, congenital anomalies, birth defects, or other medically important conditions [14]. The term "severe" is often used to describe the intensity (severity) of a medical event or an adverse reaction, as in the grading "mild", "moderate", and "severe"; thus, a severe skin reaction is not necessarily serious [14]. "Serious" and "severe" are different; SAEs are severe, but severe adverse reactions (SARs) are not necessarily serious. To improve the accuracy of the term "severe" in practical applications, SARs in this study were defined as adverse reactions requiring withdrawal of the suspected drugs and symptomatic treatment, regardless of whether the suspected drugs were used in mono- or polytherapy.

\section{Classification of SARs by drugs}

The SARs were analyzed according to the suspected AEDs. In addition, the dosage/defined daily dose (DDD) [15] was used to represent the total daily dose of the suspected drugs [16]. The DDDs of the six drugs were as follows: $300 \mathrm{mg}$ TPM and LTG, $1000 \mathrm{mg}$ CBZ and OXC, and $1500 \mathrm{mg}$ VPA and LEV [15]. If ADRs occurred when AEDs were used in polytherapy, the total daily dose of all drugs was calculated as the sum of the dosage/DDD for each drug. For example, if dizziness occurred while taking $300 \mathrm{mg} /$ day CBZ and $1000 \mathrm{mg} /$ day $\mathrm{LEV}$, the total daily dose of the two drugs was calculated as $300 / 1000+1000 / 1500=0.97$.

\section{Statistics}

Categorical/qualitative data are presented as numbers (percentages), and nonnormally distributed quantitative data are shown as the medians (ranges). The Pearson chi-square and Mann-Whitney $U$ tests were used, as appropriate. A two-sided $p<0.05$ was considered significant. A Bonferroni correction was applied for the multiple comparisons, and the $p$-values were adjusted according to the number of tests $(n=3$ for the number of AEDs received and $n=3$ for the number of AEDs related to the ADR). A corrected $p=0.0167(0.05 / 3)$ was considered significant for the number of AEDs received and the number of AEDs related to the ADR. All statistical analyses were conducted using IBM SPSS Statistics 21.0 software (IBM Corporation, NY, USA).

\section{Results}

Characteristics of epilepsy outpatients with and without ADRs

A total of 3069 epilepsy outpatients (1807 epilepsy outpatients with 5049 eligible ADRs and 1262 epilepsy outpatients without ADRs) were included. The overall ADR rate was 58.88\% (1807/3069). We compared 1807 epilepsy outpatients with ADRs and 1262 epilepsy outpatients without ADRs (Table 1). There was no significant difference in age between the two groups, regardless of the age at last visit or at epilepsy diagnosis. Compared with epilepsy outpatients without ADRs, females comprised a higher percentage of epilepsy outpatients with ADRs $(49.4 \%$ vs. $38.9 \%, p<0.001)$.

\section{General information on ADRs}

An average of 2.79 ADRs (5049/1807) occurred per patient with an ADR. In total, $53.8 \%$ of the ADRs were recorded in females and $46.2 \%$ in males, while $50.4 \%$ were caused by a single AED (Table 2).

Of the 5049 ADRs, 537 were SARs. The general profiles of non-SARs and SARs in this study is displayed in Table 2. The rates of SARs caused by one, two and three or more AEDs were 9.9, 10.0 and $19.6 \%$, respectively ( $p$ $<0.001)$. The results showed that the rate of SARs caused by three or more AEDs was significantly higher than the rate of SARs caused by one or two AEDs.

\section{ADRs and SARs by SOC}

Table 3 shows the distribution of the 5049 ADRs and 537 SARs by SOC. Altogether, 18 SOC categories were identified in this study; the top three were psychiatric disorders $(1633 / 5049,32.3 \%$ of the total ADRs), neurological disorders $(1222 / 5049,24.2 \%$ of the total ADRs) and gastrointestinal disorders $(564 / 5049,11.2 \%$ of the total ADRs). Regardless of whether the ADRs were caused by one, two, or three or more AEDs, the most commonly involved categories remained the same. There were 537 SARs (10.6\% of the total ADRs). Among these, the most common category was skin and appendage disorders, accounting for $24.4 \%(131 / 537)$.

\section{SARs by drugs}

Detailed information on the 537 SARs (including 34 SAEs) stratified by drug is shown in Additional file 1: Table S1. The top 10 most frequent SARs were rash (mainly caused by CBZ, LTG and OXC), nausea and vomiting, dizziness, increased levels of hepatic enzymes, leukopenia, somnolence, tremor, amnesia, hypoesthesia and pruritus. Only 34 SARs were SAEs, of which the following 17 were caused by a single drug: $\operatorname{CBZ}(n=2,2$ drug hypersensitivity syndrome; DHS), LTG $(n=2,1$ erythema multiforme and 1 multiple malformations), OXC $(n=1,1$ DHS), TPM $(n=9,5$ renal calculus, 1 
Table 1 Characteristics of epilepsy outpatients with and without ADRs (total = 3069)

\begin{tabular}{|c|c|c|c|}
\hline Variable & $\begin{array}{l}\text { Epilepsy outpatients with ADRs } \\
(N=1807)\end{array}$ & $\begin{array}{l}\text { Epilepsy outpatients without ADRs } \\
(N=1262)\end{array}$ & $p$-value \\
\hline Age at last visit, years $(n, \%)$ & & & $0.156^{\mathrm{a}}$ \\
\hline$\leq 12$ & $395(21.9)$ & $287(22.7)$ & \\
\hline $13-18$ & $499(27.6)$ & $323(25.6)$ & \\
\hline $19-45$ & $783(43.3)$ & $536(42.5)$ & \\
\hline$>45$ & $130(7.2)$ & $116(9.2)$ & \\
\hline Age at epilepsy diagnosis, years (median, (range)) & $18.2(0.17-86.0)$ & $18.3(0.1-81.3)$ & $0.770^{\mathrm{b}}$ \\
\hline $\operatorname{Sex}(n, \%)$ & & & $<0.001^{\mathrm{a}}$ \\
\hline Female & $893(49.4)$ & $491(38.9)$ & \\
\hline Male & $914(50.6)$ & $771(61.1)$ & \\
\hline Comorbidity (n, \%) & & & $<0.001^{\mathrm{a}}$ \\
\hline Yes & $374(20.7)$ & $180(14.3)$ & \\
\hline No & $1433(79.3)$ & $1082(85.7)$ & \\
\hline Number of AEDs received $(n, \%)^{c}$ & & & $<0.001^{\mathrm{a}}$ \\
\hline One & $489(27.1)$ & $756(59.9)$ & \\
\hline Two & $645(35.7)$ & $371(29.4)$ & \\
\hline Three+ & $673(37.2)$ & $135(10.7)$ & \\
\hline
\end{tabular}

ADRs adverse drug reactions, $A E D$ s antiepileptic drugs

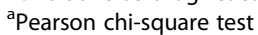

${ }^{\mathrm{b}}$ Mann-Whitney $\mathrm{U}$ test

${ }^{\mathrm{c}}$ Multiple comparison, $p=0.05 / 3=0.0167$ after Bonferroni correction. Two vs. one $p<0.001$; three+ vs. one $p<0.001$; three+ vs. two $p<0.001$

amnesia, 1 congenital spinal tumor, 1 cognitive disorder, 1 exfoliative dermatitis), and VPA ( $n=3,1$ thrombocytopenia, 1 abnormal vision, 1 DHS). The other 17 SAEs were observed with combination therapy (Additional file 1: Table S1).

\section{Discussion}

ADRs contribute significantly to patient morbidity and mortality worldwide. Both safety and tolerability are determining factors in the selection of an appropriate AED [17]. There are few observational studies on the safety of AEDs in epilepsy patients in China. In the present study, the two groups (1807 epilepsy outpatients with ADRs and 1262 epilepsy outpatients without ADRs) were compared and the data of the 5049 ADRs, which were caused by single or combinations of six commonly used AEDs, were analyzed. The results of our study provide a reference for clinicians to safely use AEDs.

Our results showed that the overall ADR rate was $58.88 \%$, which is slightly higher than the rates reported in other studies. In studies by Marson AG et al. [3] and Androsova $\mathrm{G}$ et al. [17], the ADR rates were approximately 50 and $47.6 \%$, respectively. Of the 5049 ADRs, $53.8 \%$ occurred in females. A total of 18 SOC categories were identified, and the most common ADR categories, whether resulting from a single drug or multiple drugs, were psychiatric, neurological and gastrointestinal disorders. There were 537 SARs, 34 of which were SAEs, with the majority being serious allergies, fetal malformations, renal calculus, and pancreatitis. Our study confirmed that SAEs are severe but that SARs are not necessarily serious. This distinction plays a vital role in the precise use of the terms "serious" and "severe" by clinicians.

In this study, less than $2 \%$ of the ADRs occurred in children. This low percentage may have been due to selection bias, as $95 \%$ of the WEFURD patients were adults. This study showed that ADRs were more frequent in females, and some studies on AED safety have reported similar findings [18]. Differences in ADRs due to sex may be attributed to a greater number of ADRs in females and intolerable ADRs in females. There were approximately three ADRs (2717/893) per female and $2.5(2332 / 914)$ per male. Females exhibited more SARs than non-SARs $(59.4 \%$ vs. $53.1 \%, p=0.006)$. Of the 18 SOC categories, most ADRs were categorized as psychiatric, neurological and gastrointestinal disorders, which were also the SOCs in which most ADRs to nervous system medications were found $[19,20]$. As the central nervous system (CNS) is the major action site for AEDs $[21,22]$, it is not surprising that the CNS was the system most commonly affected by AED-related ADRs [20, 23]. However, there were more ADRs categorized as psychiatric disorders, and ADRs with a frequency $>5 \%$ of the psychiatric ADRs presented as follows: amnesia $(n=492,30.13 \%)$; somnolence $(n=304$, $18.62 \%)$; insomnia ( $n=221,13.53 \%)$; anorexia $(n=197$, 
Table 2 General profiles of non-SARs and SARs in this study

\begin{tabular}{|c|c|c|c|c|}
\hline Variable & Total $(n=5049)$ & non-SARs $^{\mathrm{e}}(n=4512)$ & $\operatorname{SARs}^{\mathrm{e}}(n=537)$ & $p$-value \\
\hline Age at ADR occurrence, years (median, (range)) & $28.0(7.0-89.0)$ & $28.0(7.0-89.0)$ & $28.0(8.0-75.0)$ & $0.349^{b}$ \\
\hline $\operatorname{Sex}(n, \%)$ & & & & $0.006^{\mathrm{a}}$ \\
\hline Female & $2717(53.8)$ & $2398(53.1)$ & $319(59.4)$ & \\
\hline Male & $2332(46.2)$ & $2114(46.9)$ & $218(40.6)$ & \\
\hline Number of AEDs related to the $\mathrm{ADR}^{c}$ & & & & $<0.001^{\mathrm{a}}$ \\
\hline One $(n, \%)$ & $2546(50.4)$ & $2294(90.1)$ & $252(9.9)$ & \\
\hline Two $(n, \%)$ & $2140(42.4)$ & $1926(90.0)$ & $214(10.0)$ & \\
\hline Three+ (n, \%) & $363(7.2)$ & $292(80.4)$ & $71(19.6)$ & \\
\hline \multicolumn{5}{|l|}{$A E D s^{d}$} \\
\hline CBZ $(n, \%)$ & $1138(22.5)$ & $1000(87.9)$ & $138(12.1)$ & $0.064^{\mathrm{a}}$ \\
\hline VPA $(n, \%)$ & $2493(49.4)$ & $2228(89.4)$ & $265(10.6)$ & $0.989^{\mathrm{a}}$ \\
\hline LTG $(n, \%)$ & $1149(22.8)$ & $982(85.5)$ & $167(14.5)$ & $<0.001^{\mathrm{a}}$ \\
\hline OXC (n, \%) & $1226(24.3)$ & 1109 (90.5) & $117(9.5)$ & $0.154^{\mathrm{a}}$ \\
\hline TPM (n, \%) & $1186(23.5)$ & $1050(88.5)$ & $136(11.5)$ & $0.288^{a}$ \\
\hline LEV $(n, \%)$ & $742(14.7)$ & $664(89.5)$ & $78(10.5)$ & $0.906^{\mathrm{a}}$ \\
\hline Dosage/DDD (median, (range)) & $0.90(0.07-3.50)$ & $0.90(0.07-3.50)$ & $0.67(0.08-2.13)$ & $<0.001^{\mathrm{b}}$ \\
\hline \multicolumn{5}{|l|}{ Dosage/DDD groups } \\
\hline$\leq 0.50(n, \%)$ & $1061(21.0)$ & $907(85.5)$ & $154(14.5)$ & $<0.001^{a}$ \\
\hline $0.50-1.00(n, \%)$ & 2309 (45.7) & $2074(89.8)$ & $235(10.2)$ & $0.332^{a}$ \\
\hline $1.00-1.50(n, \%)$ & $1088(21.6)$ & $983(90.3)$ & $105(9.7)$ & $0.234^{\mathrm{a}}$ \\
\hline $1.50-2.00(n, \%)$ & $473(9.4)$ & $433(91.5)$ & $40(8.5)$ & $0.106^{\mathrm{a}}$ \\
\hline$>2.00(n, \%)$ & $118(2.3)$ & $115(97.5)$ & $3(2.5)$ & $0.004^{a}$ \\
\hline \multicolumn{5}{|l|}{ Level of causality assessment (n, \%) } \\
\hline Certain & $550(10.9)$ & $300(54.5)$ & $250(45.5)$ & $<0.001^{\mathrm{a}}$ \\
\hline Probable/Likely & $796(15.8)$ & $510(64.1)$ & $28635.9)$ & $<0.001^{\mathrm{a}}$ \\
\hline Possible & $3509(69.5)$ & $3508(99.9)$ & $1(0.1)$ & $<0.001^{a}$ \\
\hline Conditional/Unclassified & $194(3.8)$ & $194(100)$ & $0(0)$ & $<0.001^{\mathrm{a}}$ \\
\hline
\end{tabular}

ADRs adverse drug reactions, AEDs antiepileptic drugs, SARs severe adverse reactions, DDD defined daily dose

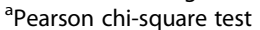

${ }^{\mathrm{b}}$ Mann-Whitney $\mathrm{U}$ test

${ }^{c}$ Multiple comparison, $p=0.05 / 3=0.0167$ after Bonferroni correction. Two vs. one $p<0.907$; three+ vs. one $p<0.001$; three+ vs. two $p<0.001$

${ }^{\mathrm{d}}$ The sum of the six AEDs was not equal to 5049 due to half of the ADRs being caused by two or three+ AEDs

eSARs refers to those ADRs requiring withdrawal of the suspected drugs and symptomatic treatment, regardless of whether the suspected drugs were used in mono- or polytherapy

$12.06 \%)$; and irritability $(n=161,9.86 \%)$, which suggests that the psychiatric symptoms caused by AEDs deserve attention from clinicians.

In our study, $10.6 \%(537 / 5049)$ of the ADRs were SARs, and $0.67 \%(34 / 5049)$ were SAEs. These results were supported by the results of a cross-sectional study in Iran [20]. Namazi, S. et al. [20] reported that almost all (99.24\%) detected ADRs to AEDs were not serious. Among the 537 SARs, we found that rash was a common reaction to CBZ, LTG, and OXC administered as single drugs; some of the rashes were serious, including DHS and erythema multiforme. The ADRs to TPM were mainly cognitive side effects, hypoesthesia and renal calculus. The ADRs to VPA were gastrointestinal disturbances and hepatotoxicity. LEV had the fewest SARs. Our findings are not novel [6, 23, 24]; current studies have shown that rashes related to AEDs are mostly idiosyncratic reactions [22]. Godhwani, N. et al. [25] reported that non-IgE-mediated reactions, more commonly associated with aromatic AEDs, can be nonspecific rashes or severe cutaneous drug reactions (SCDRs). Moreover, recent studies have demonstrated significant associations between human leukocyte antigens (HLA) and a predisposition to ADRs [26]. Ramirez, E. et al. [27] confirmed the strong association between HLA*31:01 and CBZ-drug reactions with eosinophilia and systemic symptoms (DRESS) in Europeans. The findings of studies by Chen, C. B. et al. [28] and Chong, 
Table 3 Number of adverse drug reactions and severe adverse reactions stratified by system organ class

\begin{tabular}{|c|c|c|c|c|}
\hline \multirow[t]{3}{*}{ System organ class (descending order) } & \multirow{3}{*}{$\begin{array}{l}\text { All } \\
\text { Number (severe }{ }^{a} \text { ) }\end{array}$} & \multicolumn{3}{|c|}{ Number of AEDs related to the ADR } \\
\hline & & \multirow{2}{*}{$\begin{array}{l}\text { One AEDs } \\
\text { Number (severe }{ }^{a} \text { ) }\end{array}$} & \multirow{2}{*}{$\begin{array}{l}\text { Two AEDs } \\
\text { Number (severe }{ }^{a} \text { ) }\end{array}$} & \multirow{2}{*}{$\begin{array}{l}\text { More than three AEDs } \\
\text { Number (severe }{ }^{a} \text { ) }\end{array}$} \\
\hline & & & & \\
\hline Psychiatric disorders & $1633(84)$ & $813(29)$ & 710 (39) & $110(16)$ \\
\hline Neurological disorders & $1222(93)$ & $630(46)$ & $500(37)$ & $92(10)$ \\
\hline Gastrointestinal disorders & $564(68)$ & $244(26)$ & $280(31)$ & $40(11)$ \\
\hline Skin and appendage disorders & $341(131)$ & $183(67)$ & $132(50)$ & $26(14)$ \\
\hline Body as a whole - general disorders & $260(19)$ & $137(10)$ & $108(5)$ & $15(4)$ \\
\hline Metabolic and nutritional disorders & $257(19)$ & $131(8)$ & $107(8)$ & $19(3)$ \\
\hline Liver and biliary disorders & $236(33)$ & $135(17)$ & $84(12)$ & $17(4)$ \\
\hline Vision disorders & $88(6)$ & $28(2)$ & $45(3)$ & $15(1)$ \\
\hline Reproductive disorders & $88(16)$ & $48(9)$ & $36(4)$ & $4(3)$ \\
\hline Blood disorders & $81(25)$ & $32(15)$ & $37(9)$ & $12(1)$ \\
\hline Musculoskeletal disorders & $65(11)$ & $39(5)$ & $23(4)$ & $3(2)$ \\
\hline Urinary tract disorders & $55(9)$ & $30(5)$ & $23(3)$ & $2(1)$ \\
\hline Hearing, vestibular and special senses disorders & $49(2)$ & $32(1)$ & $16(1)$ & $1(0)$ \\
\hline Cardiovascular disorders & $42(2)$ & $26(2)$ & $13(0)$ & $3(0)$ \\
\hline Vascular, bleeding and clotting disorders & $36(2)$ & $19(1)$ & $16(1)$ & $1(0)$ \\
\hline Respiratory disorders & $15(4)$ & $9(1)$ & $4(2)$ & $2(1)$ \\
\hline Immune disorders and infections & $13(9)$ & $8(6)$ & $4(3)$ & $1(0)$ \\
\hline Congenital disorders & $4(4)$ & $2(2)$ & $2(2)$ & $0(0)$ \\
\hline Total & $5049(537)$ & $2546(252)$ & $2140(214)$ & $363(71)$ \\
\hline
\end{tabular}

$A D R s$ adverse drug reactions, $A E D$ s antiepileptic drugs, SARs severe adverse reactions

aSARs refers to those ADRs requiring withdrawal of the suspected drugs and symptomatic treatment, regardless of whether the suspected drugs were used in mono- or polytherapy

K. W. et al. [29] suggested that HLA-B*15:02 is significantly associated with AED-induced SCDRs in Chinese individuals. Because of the strong genetic predisposition for certain AEDs to cause serious reactions, HLA analysis before the initiation of drug therapy is advised in certain populations [25].

Furthermore, we reported that the rate of SARs after a low treatment dose (dosage/DDD $\leq 0.50$ ) was $14.5 \%$ $(p<0.001)$. Our study also showed that more SARs occurred with multidrug treatment. The SAR rates caused by one, two and three or more AEDs were 9.9, 10.0 and $19.6 \%$, respectively $(p<0.001)$. This result suggests that clinicians should be alert for SARs, especially when three or more AEDs are used together. These findings were supported by those of other relevant studies. In the research by Horvath, L. et al. [18], the incidences of ADRs in monotherapy, biotherapy and polytherapy were $16.4,18.5$ and $23.5 \%$, respectively. The results of the study by Grundmann, M. et al. [30] showed that a higher number of drugs at supratherapeutic levels in combination therapy led to a 3 -fold higher incidence of ADRs. Studies have reported that multiple drug therapies can increase the frequency of ADRs and teratogenic risk $[31,32]$. Therefore, we suggest that the AEDs used in combination must be carefully selected [31].

\section{Conclusions}

In summary, this study was a real-world study; therefore, the results were more consistent with the clinical situation. Our findings suggest that clinicians should pay attention to psychiatric ADRs and should be alert for SARs, especially when three or more AEDs are used together. However, the confounding factors that existed when combinations of the six AEDs were used could not be estimated. Nevertheless, our study provides important reference information to guide clinicians in the safe use of AEDs. Moreover, the definition of SARs was clarified in this study, which will help clinicians accurately use the term "severe" and improve the accuracy of the monitoring data. Finally, compared with the SR system, there was a higher quality of data in this active monitoring study; only 194 ADRs (3.8\% of the total ADRs) were assessed as conditional/unclassified, with more data needed. Active surveillance might provide another method of pharmacovigilance in China. 


\section{Additional file}

Additional file 1: Table S1. Detailed information on 537 severe adverse reactions caused by drugs. Lists the severe adverse reactions to each drug, which may be found in the online version of this article. (DOCX $34 \mathrm{~kb}$ )

\section{Abbreviations}

ADRs: Adverse drug reactions; AEDs: Antiepileptic drugs;

CBZ: Carbamazepine; CFDA: China Food and Drug Administration;

CNS: Central nervous system; DDD: Defined daily dose; DHS: Drug hypersensitivity syndrome; DRESS: Drug reactions with eosinophilia and systemic symptoms; ELFURS: Epilepsy Long-term Follow Up Registry Study; FAHWMU: First Affiliated Hospital of Wenzhou Medical University; HLA: Human leukocyte antigens; LEV: Levetiracetam; LTG: Lamotrigine; OXC: Oxcarbazepine; PTs: Preferred terms; RCTs: Randomized controlled trials; SAEs: Serious adverse effects; SARs: Severe adverse reactions; SCDRs: Severe cutaneous drug reactions; SOC: System organ class; SR: Spontaneous reporting; TPM: Topiramate; VPA: Valproate; WEFURD: Wenzhou Epilepsy Follow-Up Registry Database; WHO: World Health Organization; WHOART: WHO Adverse Reaction Terminology; WHO-UMC: WHO-Uppsala Monitoring Centre

\section{Acknowledgements}

We thank all the contributing patients and Mary A., who is a Senior Editor at American Journal Experts, for editing the grammar, phrasing, and punctuation of this manuscript.

\section{Funding}

This work was funded by the National Centre for ADR Monitoring, China [Grant No. 2016(30)], and the Natural Science Foundation of Zhejiang Province [Grant No. LY15H090015]. All funders were not involved in any part of the study and have no conflicts of interest that are directly relevant to the content of this study. This study was not motivated by commercial interests.

\section{Availability of data and materials}

The datasets used and/or analyzed during the current study are available from the corresponding author on reasonable request.

\section{Authors' contributions}

YD and HX designed the study; JL, JS, SD, MY, and LW collected the data; $\mathrm{XW}, \mathrm{NX}, \mathrm{RZ}$, and $\mathrm{HC}$ corrected the data; YD and YW analyzed the data; and YD and HX prepared the manuscript. All authors contributed to and approved the final manuscript.

\section{Ethics approval and consent to participate}

We respected all patients' rights to privacy and protected their identity. All procedures performed in studies involving human participants were conducted in accordance with the ethical standards of the First Affiliated Hospital of Wenzhou Medical University Ethics Committee, and informed consent was obtained from all individual participants included in the study.

\section{Consent for publication}

Not applicable.

\section{Competing interests}

The authors declare that they have no competing interests.

\section{Publisher's note}

Springer Nature remains neutral with regard to jurisdictional claims in published maps and institutional affiliations.

\section{Author details}

'Department of Neurology, The First Affiliated Hospital of Wenzhou Medical University, Shangcai Village, Ouhai District, Wenzhou, Zhejiang Province, People's Republic of China. 'Department of Preventive Medicine, School of Public Health and Management, Wenzhou Medical University, Wenzhou, Zhejiang Province, People's Republic of China. ${ }^{3}$ Department of Psychiatry, The First Affiliated Hospital of Wenzhou Medical University, Wenzhou, Zhejiang Province, People's Republic of China.
Received: 26 September 2018 Accepted: 4 January 2019

Published online: 14 January 2019

\section{References}

1. Fricke $\mathrm{Gl}$, Jung $\mathrm{CH}$, Lerena LA, Lopez LM. Pharmacogenetics of adverse reactions to antiepileptic drugs. Neurologia. 2018;33:165-76.

2. Ye YM, Thong BY, Park HS. Hypersensitivity to antiepileptic drugs. Immunol Allergy Clin N Am. 2014;34:633-43 ix.

3. Marson AG, Al-Kharusi AM, Alwaidh M, Appleton R, Baker GA, Chadwick DW et al. The SANAD study of effectiveness of carbamazepine, gabapentin, lamotrigine, oxcarbazepine, or topiramate for treatment of partial epilepsy: an unblinded randomised controlled trial. Lancet. 2007;369:1000-15.

4. Brodie MJ, Besag F, Ettinger AB, Mula M, Gobbi G, Comai S, et al. Epilepsy, antiepileptic drugs, and aggression: an evidence-based review. Pharmacol Rev. 2016;68:563-602.

5. Schmitz B. Effects of antiepileptic drugs on mood and behavior. Epilepsia. 2006;47(Suppl 2):28-33.

6. Blaszczyk B, Lason W, Czuczwar SJ. Antiepileptic drugs and adverse skin reactions: an update. Pharmacol Rep. 2015;67:426-34.

7. Tricco AC, Cogo E, Angeliki VA, Soobiah C, Hutton B, Hemmelgarn BR, et al. Comparative safety of anti-epileptic drugs among infants and children exposed in utero or during breastfeeding: protocol for a systematic review and network meta-analysis. Systematic reviews. 2014;3:68

8. Wilby J, Kainth A, Hawkins N, Epstein D, Mclntosh H, McDaid C, et al. Clinica effectiveness, tolerability and cost-effectiveness of newer drugs for epilepsy in adults: a systematic review and economic evaluation. Health Technol Assess (Winchester, England). 2005;9:1-157 iii-iv.

9. Wong IC, Lhatoo SD. Adverse reactions to new anticonvulsant drugs. Drug Saf. 2000;23:35-56

10. Zeng QY, Fan TT, Zhu P, He RQ, Bao YX, Zheng RY, et al. Comparative longterm effectiveness of a monotherapy with five antiepileptic drugs for focal epilepsy in adult patients: a prospective cohort study. PLoS One. 2015;10:7.

11. WHO. International drug monitoring: the role of national centres. World Health Organ Tech Rep Ser. 1972;498:1-25.

12. WHO, UMC. The use of the WHO-UMC system for standardised case causality assessment. WHO. 2005. https://www.who.int/medicines/areas/ quality_safety/safety_efficacy/WHOcausality_assessment.pdf?ua=1. Accessed 10 Aug 2018.

13. WHO, UMC. International monitoring of adverse reactions to drugs: adverse reaction terminology. WHO. 2001. https://en.wikipedia.org/wiki/WHOART. Accessed 21 Aug 2018.

14. Edwards IR, Aronson JK. Adverse drug reactions: definitions, diagnosis, and management. Lancet. 2000;356:1255-9.

15. WHO Collaborating Centre for Drug Statistics Methodology. ATC/DDD Index. Norwegian Institute of Public Health. 2011. https://www.whocc.no/ atc_ddd_index/. Accessed 21 Aug 2018

16. Hao X, Chen Z, Yan B, Kwan P, Zhou D. Impact of drug manipulation on seizure freedom in adults with uncontrolled epilepsy: a prospective controlled study in rural China. CNS drugs. 2017;31:237-43.

17. Androsova G, Krause R, Borghei M, Wassenaar M, Auce $P$, Avbersek $A$, et al. Comparative effectiveness of antiepileptic drugs in patients with mesial temporal lobe epilepsy with hippocampal sclerosis. Epilepsia. 2017:58:1734-41.

18. Horvath L, Fekete K, Marton S, Fekete I. Outcome of antiepileptic drug treatment of 1282 patients with epilepsy, their pharmacovigilance reports and concomitant medication on CNS in an East-Hungarian adult database. J Neurol Sci. 2016:369:220-6.

19. Aagaard L, Strandell J, Melskens L, Petersen PS, Holme Hansen E. Global patterns of adverse drug reactions over a decade: analyses of spontaneous reports to VigiBase. Drug Saf. 2012;35:1171-82.

20. Namazi S, Borhani-Haghighi A, Karimzadeh I. Adverse reactions to antiepileptic drugs in epileptic outpatients: a cross-sectional study in Iran. Clin Neuropharmacol. 2011;34:79-83.

21. Lin JJ, Mula M, Hermann BP. Uncovering the neurobehavioural comorbidities of epilepsy over the lifespan. Lancet. 2012;380:1180-92.

22. Zaccara G, Franciotta D, Perucca E. Idiosyncratic adverse reactions to antiepileptic drugs. Epilepsia. 2007:48:1223-44

23. Kennedy GM, Lhatoo SD. CNS adverse events associated with antiepileptic drugs. CNS drugs. 2008;22:739-60.

24. Walker LE, Mirza N, Yip VLM, Marson AG, Pirmohamed M. Personalized medicine approaches in epilepsy. J Intern Med. 2015;277:218-34. 
25. Godhwani N, Bahna SL. Antiepilepsy drugs and the immune system. Ann Allergy Asthma Immunol. 2016;117:634-40.

26. Yip VL, Alfirevic A, Pirmohamed M. Genetics of immune-mediated adverse drug reactions: a comprehensive and clinical review. Clin Rev Allergy Immunol. 2015;48:165-75.

27. Ramirez E, Bellon T, Tong HY, Borobia AM, de Abajo FJ, Lerma V, et al. Significant HLA class I type associations with aromatic antiepileptic drug (AED)-induced SJS/TEN are different from those found for the same AEDinduced DRESS in the Spanish population. Pharmacol Res. 2017;115:168-78.

28. Chen CB, Hsiao YH, Wu T, Hsih MS, Tassaneeyakul W, Jorns TP, et al. Risk and association of HLA with oxcarbazepine-induced cutaneous adverse reactions in Asians. Neurology. 2017;88:78-86.

29. Chong KW, Chan DW, Cheung YB, Ching LK, Hie SL, Thomas T, et al. Association of carbamazepine-induced severe cutaneous drug reactions and HLA-B*1502 allele status, and dose and treatment duration in paediatric neurology patients in Singapore. Arch Dis Child. 2014;99:581-4.

30. Grundmann M, Koristkova B, Brozmanova H, Kacirova I. Lamotrigine drug interactions in combination therapy and the influence of therapeutic drug monitoring on clinical outcomes of adult patients. Ther Drug Monit. 2017;39:543-9.

31. Sander JW. The use of antiepileptic drugs--principles and practice. Epilepsia. 2004;45(Suppl 6):28-34.

32. Bromley R, Weston J, Adab N, Greenhalgh J, Sanniti A, McKay AJ, et al. Treatment for epilepsy in pregnancy: neurodevelopmental outcomes in the child. Cochrane Database Syst Rev. 2014;10:10.

Ready to submit your research? Choose BMC and benefit from:

- fast, convenient online submission

- thorough peer review by experienced researchers in your field

- rapid publication on acceptance

- support for research data, including large and complex data types

- gold Open Access which fosters wider collaboration and increased citations

- maximum visibility for your research: over $100 \mathrm{M}$ website views per year

At $\mathrm{BMC}$, research is always in progress.

Learn more biomedcentral.com/submissions 